\begin{tabular}{|c|l|}
\hline Title & $\begin{array}{l}\text { Photoinduced surface deformations in ion-conducting Ag-A S-S glasses. I. I sotropic deformations produced by small } \\
\text { light spots }\end{array}$ \\
\hline Author(s) & Gotoh, T.; Tanaka, K. \\
\hline Citation & $\begin{array}{l}\text { JOURNAL OF A PPLIED PHY SICS, 89(9), 4697-4702 } \\
\text { https://doi.org/L0.1063/1.1357471 }\end{array}$ \\
\hline Issue Date & 2001-05 \\
\hline Doc URL & http://hdl.handle.net/2115/5867 \\
\hline Rights & Copyright $\odot 2001$ A merican Institute of Physics \\
\hline Type & article \\
\hline File Information & JAP89-9.pdf \\
\hline
\end{tabular}

Instructions for use 


\title{
Photoinduced surface deformations in ion-conducting Ag-As-S glasses. I. Isotropic deformations produced by small light spots
}

\author{
T. Gotoh ${ }^{\mathrm{a})}$ and K. Tanaka \\ Department of Applied Physics, Faculty of Engineering, Hokkaido University, Sapporo 060-8628, Japan
}

(Received 19 December 2000; accepted for publication 29 January 2001)

\begin{abstract}
Prominent surface deformations appear in $\mathrm{Ag}-\mathrm{As}-\mathrm{S}$ chalcogenide glasses when exposed to focused laser beams. Deformation behaviors change with the beam diameter, the photon energy, the temperature at which illumination is provided, and the sample composition. In thin $\mathrm{AgAsS}_{2}$ films exposed at room temperature to a $2.0 \mathrm{eV}$ light spot with a diameter of $2 \mu \mathrm{m}$, an isotropic convex structure with a height of $\sim 0.1 \mu \mathrm{m}$ appears. The expanded region accompanies an Ag-content enhancement by $\sim 3$ at. \%, which suggests that the deformation is caused by photoinduced accumulation of $\mathrm{Ag}^{+}$ions. (C) 2001 American Institute of Physics. [DOI: 10.1063/1.1357471]
\end{abstract}

\section{INTRODUCTION}

Some kinds of atoms or ions can move easily in solids when exposed to light. A well-known example may be the photoinduced migration of $\mathrm{Ag}^{+}$ions in halide crystals, which has been applied to photographic techniques. ${ }^{1}$ It has also been demonstrated recently that several photoinduced phenomena appear in amorphous solids as well. ${ }^{2,3}$ For instance, photodoping of $\mathrm{Ag}$ into chalcogenide glasses has been discovered by Kostyshin et al. ${ }^{4}$ In contrast, in Ag$\mathrm{As}(\mathrm{Ge})-\mathrm{S}(\mathrm{Se})$ glasses with the $\mathrm{Ag}$ content greater than $\sim 30$ at. $\%, \mathrm{Ag}$ crystals are segregated to illuminated surfaces, the phenomenon being referred to as photosurface deposition (PSD) ${ }^{5}$ In addition, in glassy samples such as $\mathrm{AgAsS}_{2}, \mathrm{Ag}^{+}$ ions accumulate to illuminated regions, the phenomenon being so-called photoinduced chemical modification (PCM). ${ }^{6}$ No crystalline phases are involved here, and Ag-accumulated regions can move following illuminated regions.

In these photoinduced phenomena appearing in chalcogenide glasses, physical properties of Ag-chalcogenide glasses play decisive roles. In short, the glass can be regarded as an ion-conducting amorphous semiconductor. ${ }^{7}$ For instance, in $\mathrm{AgAsS}_{2}$ glass at room temperature, the electrical conductivity of $10^{-5} / \Omega \mathrm{cm}$ is governed by $\mathrm{Ag}^{+}$ions, ${ }^{8-10}$ and the electronic component of $10^{-8} / \Omega \mathrm{cm}$ is provided by holes. ${ }^{10}$ No evidence suggesting electron motions has been obtained. On the other hand, the optical band gap energy decreases with an increase in the $\mathrm{Ag}$ content, and in $\mathrm{AgAsS}_{2}$ it is reported to be $2.1 \mathrm{eV} .{ }^{10}$ Accordingly, when such a glass is exposed to light, electrons and holes may be photoexcited, and only holes can diffuse away, which gives rise to a Dember voltage, i.e., the illuminated region being negatively charged. In response, $\mathrm{Ag}^{+}$ions in unilluminated regions will migrate toward the illuminated region. This response can be regarded as a kind of photoelectro-ionic processes, which is assumed to be an essential mechanism of the photodoping, PSD, and PCM phenomena. ${ }^{2-7}$ Note that most studies on these phenomena had employed unpolarized light. ${ }^{2}$

\footnotetext{
${ }^{a)}$ Electronic mail: tgotoh@eng.hokudai.ac.jp
}

Recently, Tanaka et al. have demonstrated that an anisotropic macroscopic deformation occurs in $\mathrm{Ag}-\mathrm{As}(\mathrm{Ge})-\mathrm{S}$ films when exposed to linearly polarized light spots, ${ }^{11}$ while the formation mechanism remains to be speculative. ${ }^{12}$ In the present and the following work (called article II), therefore, deformation characteristics are investigated in detail, and the mechanisms are considered. Surprisingly, it has been discovered that, depending upon the size of light spots, the deformation becomes isotropic (for the spot size smaller than $\sim 5$ $\mu \mathrm{m})$ and anisotropic (for the size greater than $\sim 5 \mu \mathrm{m}$ ). Since the isotropic deformation seems to arise from simpler processes, we will investigate the feature in the present work. Then, in article II, we will consider the origin of the anisotropic pattern formation. These studies will show that the surface deformation in Ag-chalcogenide glasses occurs through the photoelectro-ionic interaction which is inherent to ion-conducting amorphous semiconductors.

\section{EXPERIMENT}

Two types of $\mathrm{Ag}-\mathrm{As}-\mathrm{S}$ glasses were employed in the present study. The first were $\mathrm{AgAsS}_{2}$ films with a thickness of $\sim 1 \mu \mathrm{m}$, which had been prepared through photodoping process, ${ }^{2}$ the details being described elsewhere. ${ }^{6}$ In brief, appropriate amounts of $\mathrm{Ag}$ and $\mathrm{AsS}_{2}$ were successively evaporated onto glass slides, and the bilayer films were exposed to light emitted from a Xe lamp until the photodoping was completed. The second were melt-quenched $\mathrm{Ag}_{2} \mathrm{~S}-\mathrm{As}_{2} \mathrm{~S}_{3}$ glasses with $\mathrm{Ag}$ contents of $0-35$ at. \%, which were employed in the previous compositional study. ${ }^{10}$ The glass ingots were polished to disks with a thickness of $\sim 0.2 \mathrm{~mm}$, and then, were annealed at the glass transition temperatures for stabilization.

These samples were illuminated at 4-300 K with light emitted from two continuous wave lasers: a $\mathrm{He}-\mathrm{Ne}$ laser $(\hbar \omega=2.0 \mathrm{eV})$ and a solid-state laser $(\hbar \omega=2.6 \mathrm{eV})$. The laser light was focused to spots with diameters of 2-5 $\mu \mathrm{m}$ using microscope objective lenses. In some cases such as low-temperature illumination, focused light was transmitted to sample surfaces using optical fibers with core diameters of 
3 and $6 \mu \mathrm{m}$. These light beams were linearly polarized, unless otherwise stated. To obtain smaller light spots, we utilized tapered fiber probes which were commercially available for a scanning near-field optical microscope (SNOM). Aperture size of the probes was reported to be $0.15 \mu \mathrm{m},{ }^{13}$ while it was not known if the polarization was retained for emitted light. Gap distances between the probe and a sample surface were controlled using an atomic force mode in the SNOM. ${ }^{13}$ Intensities of these laser beams were adjusted using neutral density filters.

Produced deformations were analyzed employing three pieces of equipment at room temperature. The shape of photoinduced deformations was inspected using a Nomarskitype interference optical microscope, and the profile was measured with an atomic force microscope (AFM). In addition, atomic compositions were evaluated using an electron probe microanalyzer (EPMA). Since $\mathrm{Ag}^{+}$ions are liable to migrate under electron-beam exposures, ${ }^{14}$ the probe condition was carefully selected so that electron-beam exposures gave no appreciable effects, while retaining an acceptable signal-to-noise level. The condition was, typically, an acceleration voltage of $10 \mathrm{kV}$ and an electron current of $600 \mathrm{pA}$, and characteristic $\mathrm{x}$-ray intensities emitted from rasterscanned sample areas of $10 \mu \mathrm{m}^{2}$ were counted with a measuring time of $10 \mathrm{~s}$.

\section{RESULTS AND DISCUSSION}

\section{A. Overall behaviors}

Figure 1(a) shows an AFM image of a $1-\mu$ m-thick $\mathrm{AgAsS}_{2}$ film which has been exposed to a focused $\mathrm{He}-\mathrm{Ne}$ laser beam at room temperature. The power density estimated from a light intensity of $0.2 \mathrm{~mW}$ and a beam diameter of $\sim 2 \mu \mathrm{m}$ is $\sim 6 \mathrm{~kW} / \mathrm{cm}^{2}$, and the exposure time is $2 \mathrm{~min}$. We see in the illuminated region a circular convex structure, the peripheral being slightly hollowed. Height of the deformation measured from the initial surface level is $\sim 120 \mathrm{~nm}$, which corresponds to $\sim 12 \%$ of the sample thickness. Diameter of the expansion is $\sim 2 \mu \mathrm{m}$, being nearly the same in size with the light spot. In this example the exposure is provided from the free surface of the film, and it has been demonstrated that exposures from the substrate side produce similar deformations. Note that, although the laser light is linearly polarized, the polarized direction does not give any traces upon the deformation shape.

To examine the behavior produced by smaller light spots, we have employed the SNOM probes. Figure 1(b) shows a typical deformation obtained using a $0.15 \mu \mathrm{m}$ aperture probe and the $2.6 \mathrm{eV}$ light source. The light fed into the fiber had an intensity of $100 \mathrm{nW}$, and an output intensity from the probe was estimated at $\sim 1 \mathrm{nW} .{ }^{13}$ With an exposure to the light for $1 \mathrm{~min}$, a convex deformation appears, which is double structured, the central expansion with a height of 5 $\mathrm{nm}$ and a diameter of $0.3 \mu \mathrm{m}$, and a wider expansion with a diameter of $\sim 1.3 \mu \mathrm{m}$. Exposures of $2.0 \mathrm{eV}$ have also produced similar convex deformations. We, therefore, can assume that the isotropic convex deformation is produced in $\mathrm{AgAsS}_{2}$ films when exposed to small light spots.
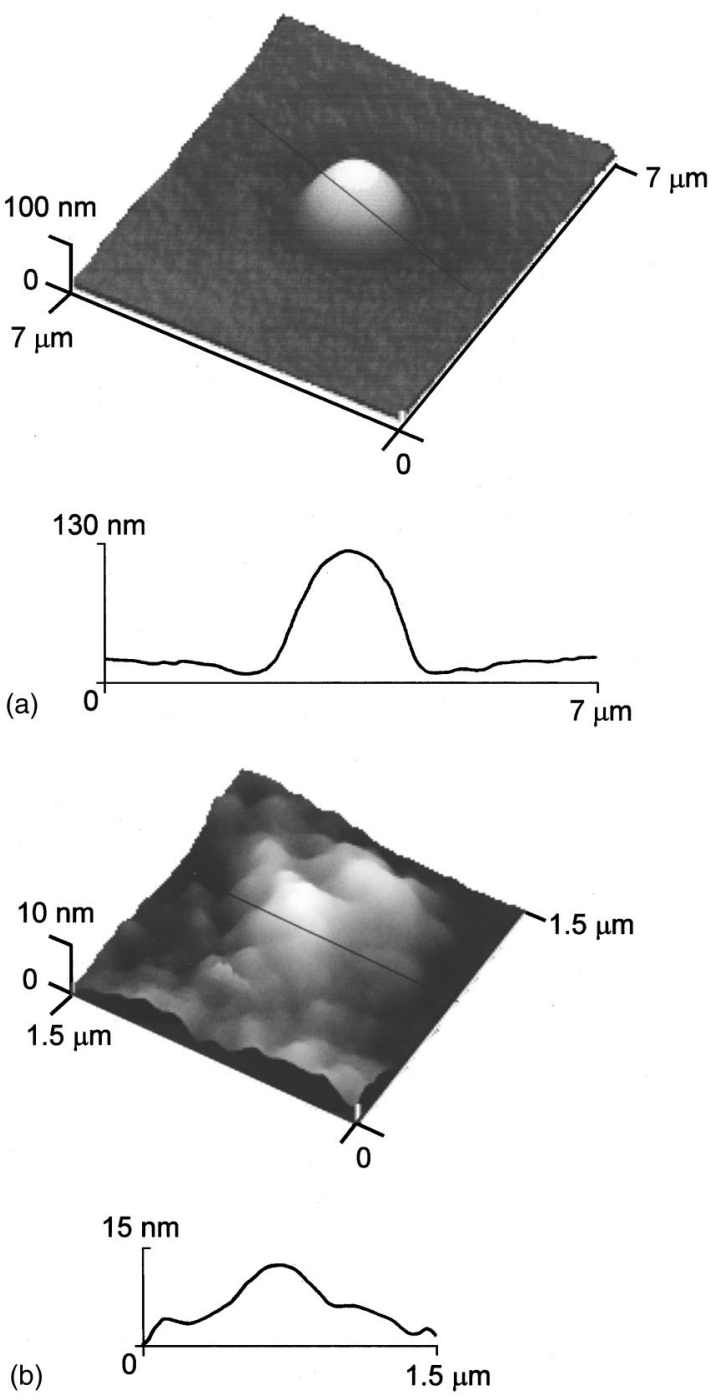

FIG. 1. AFM images of $\mathrm{AgAsS}_{2}$ films (a) after an exposure for 2 min to 2.0 $\mathrm{eV}$ light and (b) an exposure for $1 \mathrm{~min}$ to $2.6 \mathrm{eV}$ near-field light. The lower traces show the cross-sectional views along the solid lines in the images.

Thermal stability of the deformation was investigated. It was found that the produced deformation is stable at room temperature in the dark, at least, over a half year. On the other hand, the deformation could be erased with an annealing treatment at $420 \mathrm{~K}$, which is the glass transition temperature of $\mathrm{AgAsS}_{2},{ }^{9}$ for $1 \mathrm{~h}$ in a flowing Ar gas. This result suggests that the photoinduced crystallization, ${ }^{3}$ which cannot be reversed by annealing, is not responsible for this phenomenon.

It is known that $\mathrm{Ag}^{+}$ions in $\mathrm{AgAsS}_{2}$ glass are likely to move toward illuminated regions, ${ }^{5-7}$ and hence compositional evaluations have been carried out using the EPMA. Then, the Ag content in the convex region has been found to increase by $\sim 3$ at. $\%$ with illumination. Note that, since the spatial resolution of the composition analysis $(\sim 3 \mu \mathrm{m}$ in length) is comparable with the deformation diameter [Fig. 1(a)], a maximal compositional change may be greater. In contrast to the Ag enhancement, no compositional changes have been detected for As and $\mathrm{S}$ atoms. These results suggest that $\mathrm{Ag}^{+}$ions with a density of $\sim 10^{21} \mathrm{~cm}^{-3}(\sim 3$ at. \%) migrate into the convex region with illumination. 


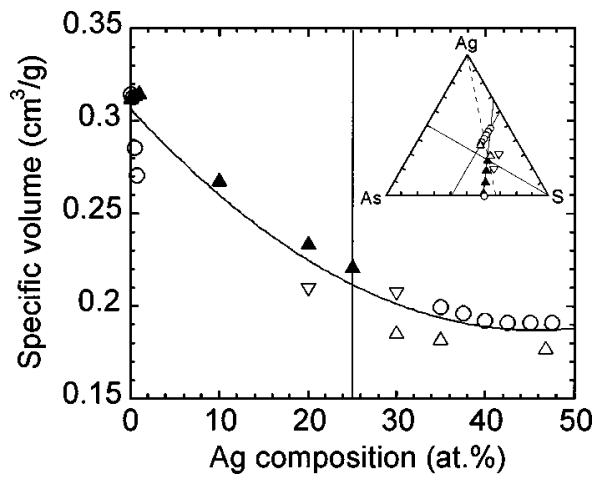

FIG. 2. The specific volume of $\mathrm{Ag}-\mathrm{As}-\mathrm{S}$ glasses as a function of the $\mathrm{Ag}$ content. The data are obtained from Refs. 8 and 15, and the solid line shows a least-squares fit to all the data points. The symbols are defined in the inset, which shows the actual glass compositions. The PCM process in $\mathrm{AgAsS}_{2}$ is assumed to occur along the dotted line in the inset.

\section{B. Proposed mechanism}

Ag-chalcogenide glasses are known to exhibit the PCM phenomenon, ${ }^{6}$ and the Ag-content increase described above implies that the convex deformation occurs as a result of the PCM. The PCM process is assumed to proceed as follows: ${ }^{6}$ When an Ag-chalcogenide glass is exposed to band gap light, electrons and holes are photoexcited, and the holes diffuse toward unilluminated regions, since the hole is more mobile. The illuminated region then becomes negatively charged, and in response, $\mathrm{Ag}^{+}$ions in the unilluminated region migrate toward the illuminated region. This migration gives an $\mathrm{Ag}$ concentration increase of $\sim 5$ at. $\%$ in $\mathrm{AgAsS}_{2}$, which is a similar level to the present result. Until now, however, no macroscopic deformations have been reported for the PCM.

Actually, if the Ag-content increase can give rise to a volume expansion is a subtle problem. The atomic volume data ${ }^{8,15}$ shown in Fig. 2 imply that the amorphous structure becomes more compact with the Ag content, which may provide a volume reduction. However, in the PCM process, since $\mathrm{Ag}^{+}$ions are injected into an illuminated region, the region becomes heavier. In consequence, the volume change is determined as a result of the structural compaction and the mass increase. Then, a simple analysis described in Ref. 16 suggests that the Ag-content increase of 3-5 at. \% gives rise to a volume expansion of $\sim 4 \%$. This magnitude is smaller than the experimental observation of 12\% shown in Fig. 1(a), while the difference can be ascribed to different amorphous structures between the photoproduced (ion injected) and the melt-quenched (relaxed) glass. We also note that the peripheral groove shown in Fig. 1(a) can be accounted for as a manifestation of the Ag depletion. The distance between the groove and the convex structure is $\sim 1 \mu \mathrm{m}$, which probably reflects the diffusion length of holes or the migration distance of $\mathrm{Ag}^{+}$ions. In the following, we will examine if this PCM model can explain other features observed under various experimental conditions.

\section{Time and intensity dependence}

Figure 3 shows the exposure-time dependence of the deformation height as a function of light intensities. For $2.0 \mathrm{eV}$

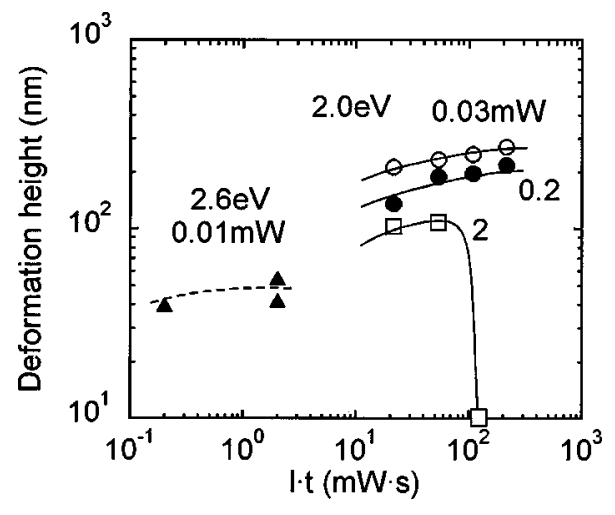

FIG. 3. The deformation height in $\mathrm{AgAsS}_{2}$ films as functions of the photon energy, the light intensity, and the exposure dose. The spot diameter is fixed at $2 \mu \mathrm{m}$.

light the light intensity is varied over two orders of magnitudes, while the three curves show similar increases when exposures are started. Interestingly, weaker light gives a greater deformation. Under weaker illumination, the temperature rise of illuminated regions is assumed to be smaller, and accordingly, this feature suggests that thermal effects are not essential to the convex-pattern formation. This result is, therefore, consistent with the PCM model assuming the photoelectro-ionic interaction.

In contrast, we see in Fig. 3 that $2 \mathrm{~mW}$ illumination gives a height decrease at around $50 \mathrm{~s}$. This decrease has resulted in concave deformations, i.e., circular holes, which seem to arise from sample vaporization. Actually, a theoretical analysis suggests that the $2 \mathrm{~mW}$ illumination can give rise to a temperature rise of $\sim 10^{2} \mathrm{~K} .{ }^{17}$

When the light intensity is intermediate, we can envisage that the photoinduced $\mathrm{Ag}^{+}$accumulation is suppressed by outward thermal diffusion. Quantitatively, at $0.2 \mathrm{~mW}$, the temperature rise is estimated at $10^{1} \mathrm{~K} .{ }^{17}$ On the other hand, the thermal diffusion coefficient of $\mathrm{Ag}^{+}$ions in $\mathrm{AgAsS}_{2}$ is reported to be, e.g., at $350 \mathrm{~K}, 2 \times 10^{-10} \mathrm{~cm}^{2} / \mathrm{s}$ (Fig. 5), ${ }^{10}$ and then such a temperature rise can cause $\mathrm{Ag}^{+}$ion diffusion with a distance of $\sim 10^{0} \mu \mathrm{m}$ for $10^{2} \mathrm{~s}^{18}$ This length is comparable with the size of the light spot employed here. It seems possible to conclude, therefore, that illumination with moderate intensities suppresses the photoelectro-ionic volume expansion through the outward thermal diffusion of $\mathrm{Ag}^{+}$ions.

\section{Spectral and volume effects}

Figure 3 includes a time dependence of the deformation height by $2.6 \mathrm{eV}$ light of $10 \mu \mathrm{W}$. We note that, in the intensity range of $0.1-10 \mu \mathrm{W}$, saturated heights have been roughly the same, which suggests that the thermal effects can be neglected. However, more intense illumination has produced circular holes on the sample, which is probably due to the thermal evaporation.

Comparing the characteristics obtained by 2.0 and 2.6 $\mathrm{eV}$ light (Fig. 3), we can point out two quantitative differences. First, the deformation height produced by $2.6 \mathrm{eV}$ is approximately half of that by $2.0 \mathrm{eV}$. Second, $2.6 \mathrm{eV}$ light gives faster changes. 


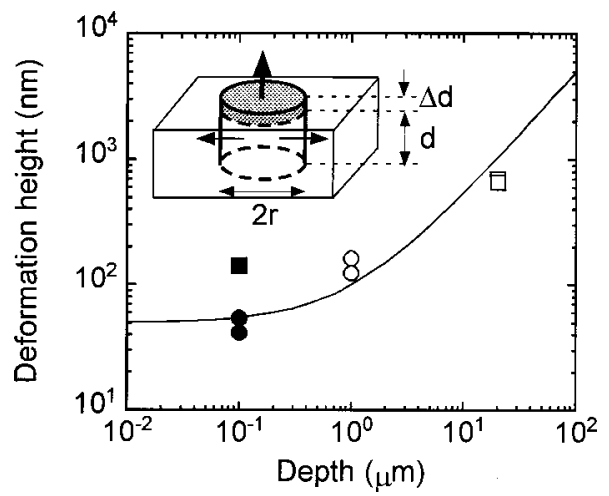

FIG. 4. The deformation height as a function of the depth of illuminated regions in $\mathrm{AgAsS}_{2}$ films and bulk samples exposed to light spots with a diameter of $2 \mu \mathrm{m}$. Open symbols and closed symbols are the data obtained by exposures to 2.0 and $2.6 \mathrm{eV}$ light, and circles and squares show the data obtained in 1- $\mu$ m-thick and 0.2 -mm-thick samples. The inset illustrates the expansion of an illuminated volume.

These differences can be understood as follows: The optical band gap energy of $\mathrm{AgAsS}_{2}$ glass is reported to be $\sim 2.1$ $\mathrm{eV},{ }^{10}$ and accordingly, both 2.0 and $2.6 \mathrm{eV}$ beams can excite electrons and holes. However, the penetration depths (the inverse of absorption coefficients) of 2.0 and $2.6 \mathrm{eV}$ light are estimated at 20 and $0.1 \mu \mathrm{m},{ }^{10}$ which are greater and smaller than the sample thickness of $\sim 1 \mu \mathrm{m}$. Hence, $2.0 \mathrm{eV}$ exposures can increase the Ag content uniformly throughout the sample thickness, while $2.6 \mathrm{eV}$ exposures can give rise to the $\mathrm{Ag}$ accumulation only in the surface region of $\sim 0.1 \mu \mathrm{m}$. As a result, the deformation induced by $2.0 \mathrm{eV}$ light becomes seemingly greater. On the other hand, the deformation occurs faster for $2.6 \mathrm{eV}$ light due to a higher absorption coefficient, ${ }^{10}$ which gives more absorbed photons. Actually, if we evaluate the deformation as a function of absorbed photon numbers, the rate for $2.6 \mathrm{eV}$ light becomes similar to that for $2.0 \mathrm{eV}$.

So far it has been asserted that the motive force of the convex deformation in $\mathrm{AgAsS}_{2}$ films is the photoinduced $\mathrm{Ag}^{+}$ion accumulation, while it should be underlined that the motive force alone cannot produce the observed expansion. For the macroscopic volume expansion to appear, amorphous networks should become viscous.

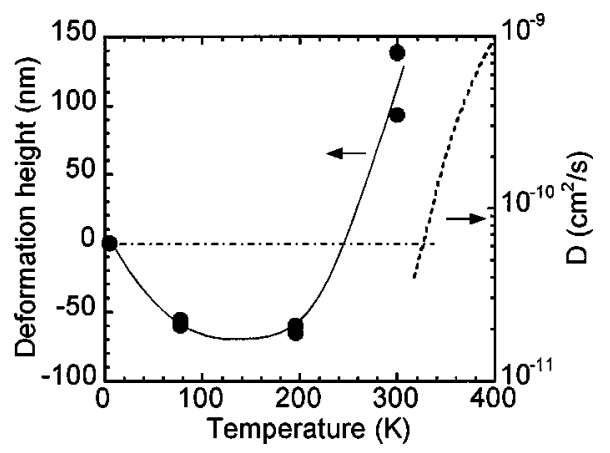

FIG. 5. Temperature dependence of the deformation height in an $\mathrm{AgAsS}_{2}$ film upon $0.2 \mathrm{~mW} \mathrm{He}-\mathrm{Ne}$ laser illumination. The light spot is fixed at $4 \mu \mathrm{m}$ in diameter. Exposure times are 2-30 min, which seem to give saturated values. The diffusion coefficient of $\mathrm{Ag}^{+}$ions in $\mathrm{AgAsS}_{2}$ glass is also shown by a dotted line (see Ref. 8).

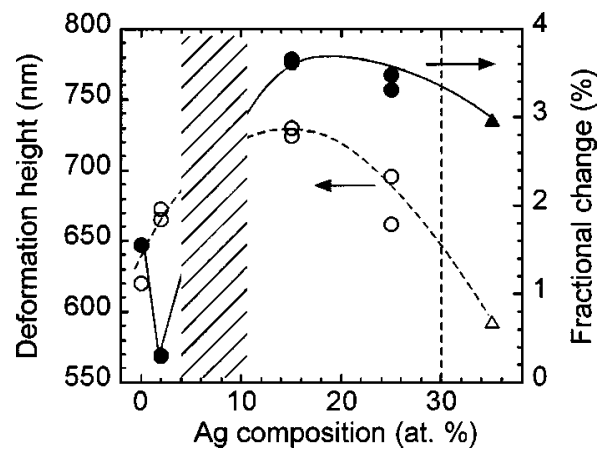

FIG. 6. The composition dependence of the deformation height (open symbols with the left-hand axis) and the fractional change (closed symbols with the right-hand axis) in the $\mathrm{As}_{2} \mathrm{~S}_{3}-\mathrm{Ag}_{2} \mathrm{~S}$ system as a function of the $\mathrm{Ag}$ content. Bulk samples are exposed for 2 min to a $2.0 \mathrm{eV}$ light beam with an intensity of $1 \mathrm{~mW}$ and a spot diameter of $\sim 3 \mu \mathrm{m}$. The slanted region shows the composition, where the glass formation is difficult (see Ref. 9).

Actually, Fig. 4 manifests such an effect. Here, the height of deformations induced by 2.0 (open symbols) and $2.6 \mathrm{eV}$ (solid symbols) light in films (circles) and bulk samples (squares) is plotted as a function of the depth of illuminated regions. The depth is equated to the penetration depth of light when it is smaller than sample thickness, and to the sample thickness when the penetration depth is greater than that. The light-spot diameter is fixed at $2 \mu \mathrm{m}$.

We can expect a volume effect here. That is, if the glass network is viscous under illumination, i.e., if the photoinduced fluidity appears, ${ }^{19}$ the cylindrical volume (see inset in Fig. 4) defined by the penetration depth and the spot size tends to expand, and an expansion can appear only at the free surface due to the existence of peripheral rigid regions. Such a volume expansion can be phenomenologically analyzed in a simple way, ${ }^{20}$ and the result obtained after the least-squares fitting is shown by a solid curve in Fig. 4. We see that the fitting is satisfactory, which demonstrates the importance of the volume effect. Note, however, that microscopic mechanisms of the photoinduced fluidity in amorphous networks remain to be studied. ${ }^{19}$

\section{E. Temperature dependence}

Dependence of the photoinduced deformation upon the temperature at which exposures are provided has been investigated using an $\mathrm{AgAsS}_{2}$ film and a $0.2 \mathrm{~mW} \mathrm{He}-\mathrm{Ne}$ laser beam. Figure 5 shows the result, together with the diffusion coefficient of $\mathrm{Ag}$ in $\mathrm{AgAsS}{ }_{2}$ glass. ${ }^{8}$ At $4 \mathrm{~K}$, no deformation has been formed, which is consistent with negligible ionic motions at low temperatures. However, surprisingly, illumination at 80 and $200 \mathrm{~K}$ has produced clear "concave" deformations, where no appreciable thermal diffusion of $\mathrm{Ag}$ can be expected from the diffusion coefficient. These observations may be experimental artifacts arising from the deformation measurements made at room temperature. Or, we may speculate the diffusion of electrons at low temperatures which can induce ionic motions. Further studies are needed for these anomalous results. At $300 \mathrm{~K}$, convex deformations appear, which is consistent with the PCM model. 

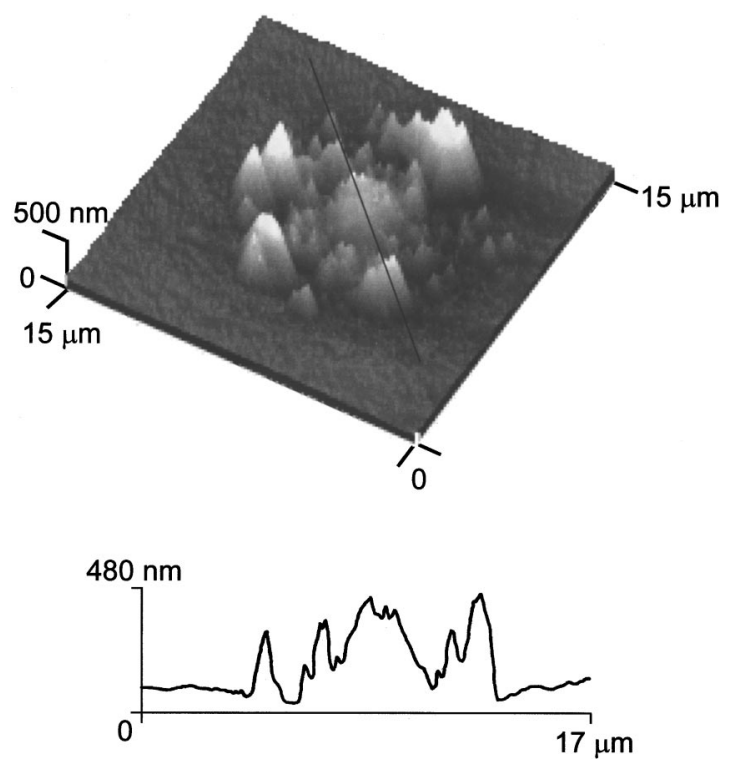

FIG. 7. An AFM image of an $\mathrm{Ag}_{35} \mathrm{As}_{19} \mathrm{~S}_{46}$ film after an exposure for $2 \mathrm{~min}$ to $2.0 \mathrm{eV}$ light with a spot diameter of $2 \mu \mathrm{m}$. The lower portion shows a cross-sectional view along the solid line in the image.

\section{F. Composition dependence}

Figure 6 shows the composition dependence of the photoinduced deformation in the $\mathrm{As}_{2} \mathrm{~S}_{3}-\mathrm{Ag}_{2} \mathrm{~S}$ glass system, in which the $\mathrm{Ag}$ content is varied at $0-35$ at. \%. Note that bulk samples are employed here, since the photodoping process cannot produce homogeneous films with varied $\mathrm{Ag}$ contents. $^{2}$ Also note that the glass formation is difficult at the Ag concentrations of $4-10$ at. $\%$ and higher than 40 at. \%. ${ }^{9}$ Here, the samples with a thickness of $\sim 0.2 \mathrm{~mm}$ are irradiated by a $2.0 \mathrm{eV}$ beam under the same exposure condition. However, since the absorption coefficient at $2.0 \mathrm{eV}$ changes widely with the $\mathrm{Ag}$ content, when evaluating the fractional height change (solid line), the effective thickness is equated to the sample thickness (for the compositions of 0 and 2 at. \%) or to the penetration depths (for other compositions). It is known that three kinds of phenomena appear in this glass system.

In $\mathrm{As}_{2} \mathrm{~S}_{3}$, a simple volume expansion accompanying nonperipheral grooves appears upon intense subband gap illumination, the phenomenon being referred to as the giant photoexpansion. ${ }^{21}$ In short, the mechanism is ascribed to intrinsic photostructural expansion of chalcogenide networks and the photoinduced fluidity, although the details remain to be studied. ${ }^{19,21}$ Then, a clear minimum existing at 2 at. $\%$ in the fractional change in Fig. 6 strongly suggests that this photoexpansion mechanism is not responsible for the $\mathrm{Ag}$ chalcogenide glasses.

Alternatively, the existence of the minimum is consistent with the deformation mechanism inherent to $\mathrm{Ag}$ chalcogenide glasses. In the composition region of 15-25 at. $\%$, a broad maximum seems to exist in the fractional change, which is consistent with a thermodynamic model adapted for the PCM. ${ }^{6}$ That is, an effective free energy of this system is assumed to be minimal at $\sim 25 \mathrm{Ag}$ at. $\%,{ }^{22}$ and accordingly, Ag-content modifications can occur relatively easily around this composition.

Last, in the samples with the $\mathrm{Ag}$ content greater than $\sim 30$ at. $\%$, the PSD phenomenon appears. ${ }^{2,5}$ Actually, as shown in Fig. 7, $2.0 \mathrm{eV}$ light with a spot diameter of $2 \mu \mathrm{m}$ has produced irregular extrusions, which are probably $\mathrm{Ag}$ crystallites. Here, it is interesting to note that the extrusions distribute more widely $(\sim 10 \mu \mathrm{m})$ than the spot size. The difference may represent the diffusion length of holes. ${ }^{5,7}$

\section{SUMMARIES}

It has been demonstrated that, in the ion-conducting amorphous semiconductor $\mathrm{Ag}-\mathrm{As}-\mathrm{S}$, light illumination can produce volume expansions. The expansion is considered to be a manifestation of the photoinduced chemical modification, ${ }^{6}$ which is a kind of photoelectro-ionic phenomena. That is, the diffusion of photoexcited holes makes the illuminated region negatively charged, which promotes the accumulation of $\mathrm{Ag}^{+}$ions, and then the volume expansion occurs.

In applications, the photoinduced volume-expansion phenomenon is promising for the direct production of a variety of optical elements such as relief gratings and microlense.

${ }^{1}$ J. F. Hamilton, Adv. Phys. 37, 359 (1988).

${ }^{2}$ A. V. Kolobov and S. R. Elliott, Adv. Phys. 40, 625 (1991).

${ }^{3}$ M. Frumar, Z. Polak, Z. Cernosek, B. Frumarova, and T. Wagner, Chem. Papers 51, 310 (1997).

${ }^{4}$ S. M. T. Kostyshin, E. U. Mikhailovskya, and P. F. Romanenko, Sov. Phys. Solid State 8, 451 (1966).

${ }^{5}$ S. Maruno and T. Kawaguchi, J. Appl. Phys. 46, 5312 (1975); 77, 628 (1995); T. Kawaguchi, S. Maruno, and S. R. Elliott, J. Non-Cryst. Solids 202, 107 (1996).

${ }^{6}$ K. Tanaka, N. Yoshida, and Y. Yamaoka, Philos. Mag. Lett. 68, 81 (1993); N. Yoshida and K. Tanaka, J. Appl. Phys. 78, 1745 (1995).

${ }^{7}$ K. Tanaka, J. Non-Cryst. Solids 164-166, 1179 (1993).

${ }^{8}$ Y. Kawamoto and M. Nishida, Phys. Chem. Glasses 18, 19 (1977).

${ }^{9}$ Z. U. Borisova, Glassy Semiconductors (Plenum, New York, 1981).

${ }^{10}$ M. Ohto, M. Itoh, and K. Tanaka, J. Appl. Phys. 77, 1034 (1995).

${ }^{11}$ K. Tanaka, T. Gotoh, and H. Hayakawa, Appl. Phys. Lett. 75, 2256 (1999)

${ }^{12}$ T. Gotoh, H. Hayakawa, and K. Tanaka, J. Non-Cryst. Solids 266-269, 944 (2000).

${ }^{13}$ Data sheets of SNOM (Seiko Instruments Inc.).

${ }^{14}$ N. Yoshida and K. Tanaka, J. Non-Cryst. Solids 210, 119 (1997).

${ }^{15}$ T. Kawaguchi, S. Maruno, and S. R. Elliott, J. Non-Cryst. Solids 211, 187 (1997).

${ }^{16}$ This magnitude is obtained as follows: A simple calculation shows $\Delta V / V=\{(d v / d x) / v+m(\mathrm{Ag}) / m\} \Delta x$, where $\Delta V / V$ is the fractional volume change of an illuminated region, $v$ the specific volume, $x$ the fractional atomic content of $\mathrm{Ag}, m(\mathrm{Ag})$ the atomic weight of $\mathrm{Ag}$, and $m$ the averaged atomic weight for the initial composition. In this equation, the first term of the right-hand side reflects some structural change with the $\mathrm{Ag}$ content, and the second term represents the volume increase of the illuminated region with the $\mathrm{Ag}$ accumulation. Figure 2 implies that the first term is negative, which means that the amorphous structure becomes compact with $x$, while the second term is always positive. Quantitatively, using the solid line in Fig. 2, we obtain $\Delta V / V \sim \Delta x$ at around $x=0.25$, i.e., the second term prevails in the volume change.

${ }^{17} \mathrm{~A}$ theoretical formula $\Delta T=\alpha d I / \kappa r$ is employed here, where $\alpha(=400$ $\mathrm{cm}^{-1}$ ) is the absorption coefficient of the film, $d$ the film thickness, $I$ the light intensity, $\kappa(=0.02 \mathrm{~W} / \mathrm{cm} \mathrm{K})$ the thermal conductivity of the substrate glass, and $r$ the radius of light spots. 
${ }^{18}$ The diffusion length of $\mathrm{Ag}^{+}$ions is estimated by $(D t)^{1 / 2}$, where $D$ is the diffusion coefficient and $t$ is a relevant time.

${ }^{19}$ H. Hisakuni and K. Tanaka, Science 270, 974 (1995); K. Tanaka, J. NonCryst. Solids 266-269, 889 (2000).

${ }^{20}$ The line gives the effective expansion $\Delta d\left(=\Delta d_{0}(1+d / r)\right.$ ) (see Ref. 21$)$, which is obtained by assuming that the illuminated cylindrical volume with a radius of $r$ and a depth of $d$ possesses an intrinsic expansion of $\Delta d_{0}$. From the fit, we obtain $\Delta d_{0} / d=0.05$.

${ }^{21}$ H. Hisakuni and K. Tanaka, Appl. Phys. Lett. 65, 2925 (1994); K. Tanaka, Phys. Rev. B 57, 5163 (1998).

${ }^{22}$ A. E. Owen, A. P. Firth, and P. J. S. Ewen, Philos. Mag. B 52, 347 (1985). 